\title{
Pratiques
}

Linguistique, littérature, didactique

\section{Pour un dialogue interdidactique français/arts}

\section{Anne Leclaire-Halté et André Petitjean}

\section{(2) OpenEdition}

\section{Journals}

Édition électronique

URL : http://journals.openedition.org/pratiques/3553

DOI : 10.4000/pratiques.3553

ISSN : 2425-2042

\section{Éditeur}

Centre de recherche sur les médiations (CREM)

\section{Référence électronique}

Anne Leclaire-Halté et André Petitjean, «Pour un dialogue interdidactique français/arts », Pratiques [En ligne], 175-176 | 2017, mis en ligne le 22 décembre 2017, consulté le 21 décembre 2020. URL : http:// journals.openedition.org/pratiques/3553; DOI : https://doi.org/10.4000/pratiques.3553

Ce document a été généré automatiquement le 21 décembre 2020.

(c) Tous droits réservés 


\title{
Pour un dialogue interdidactique français/arts
}

\author{
Anne Leclaire-Halté et André Petitjean
}

1 Il semble loin le temps des années 60-70 quand les textes officiels donnaient pour objectif principal à l'enseignement des Lettres l'étude des œuvres littéraires relevant du seul patrimoine hexagonal. À cette époque, le cloisonnement disciplinaire, à la fois «encyclopédique» et "additif», était associé à un mode de travail pédagogique essentiellement transmissif (Lenoir \& Sauve, 1998). Face à cette doxa dominante, rares étaient les expériences pédagogiques interdisciplinaires que promurent l'Institut national de recherche pédagogique (INRP) et les activités d'éveil (Best, 1973), les mouvements pédagogiques comme le Cercle de recherche et d'action pédagogiques (Crap) (Cahiers pédagogiques, $\left.\mathrm{n}^{\circ} 97,123,148-149,166\right)$ ou les revues pédagogiques. À titre d'exemples, nous renvoyons à différents numéros des revues Le Français aujourd'hui ( ${ }^{\circ}$ $45,53,56,59,74)$ et Pratiques ( $n^{\circ} 31$ et 36$)$. Si l'interdisciplinarité a connu son heure de gloire dans le monde anglo-saxon des années 70, elle n'a été que partiellement introduite dans le système scolaire français avec les textes officiels de 1977 pour les collèges. En effet, les programmes successifs du secondaire et, à un degré moindre, du primaire, sont révélateurs d'une tension entre la volonté d'intégrer une perspective interdisciplinaire et le maintien d'une structuration disciplinaire et d'un cloisonnement des savoirs. Et pourtant, on ne manque pas de rapports officiels qui défendent et illustrent l'intérêt de l'interdisciplinarité (Legrand, 1981' ; Bourdieu \& Gros, 1989)². Ils déclenchèrent l'ire des antipédagogues tels que M. Maschino (1984), D. de La Martinière (1984), A. Finkielkraut (1987), J.-M. Domenach (1989). D'autres rapports suivirent (Morin, 19983 ; Meirieu, 1998) si bien que les textes officiels finirent, même partiellement, par reconnaitre la nécessité de travailler "autrement", par l'intermédiaire de dispositifs divers («travaux croisés ", «itinéraires de découvertes ", " parcours diversifiés », « travaux personnels encadrés ») $)^{5}$. Il aura surtout fallu attendre le décret sur le Socle commun de connaissances et de compétences (MEN, 2006), revu et approfondi par le décret sur le Socle commun de connaissances, de compétences et de culture (MEN, 2015a). Ils modifient l'organisation de la scolarité obligatoire sous la forme de différents cycles et proposent l'un sept compétences ${ }^{6}$ et l'autre cinq domaines ${ }^{7}$. Il 
ressort de ces derniers textes qu'est préconisée, pour nous en tenir à la problématique du présent numéro de Pratiques, la mise en dialogue de l'enseignement de la littérature avec les arts. Cela apparait clairement avec le décret de 2006 dans lequel « la culture humaniste » s'appuie sur « la fréquentation des œuvres littéraires » et « se nourrit des apports de l'éducation artistique ». On recommande aussi de placer les élèves dans des rapports productifs par rapport aux différentes pratiques artistiques.

2 À quoi il faut ajouter, avec le décret de 2008 consacré à l'organisation de l'enseignement de l'histoire des arts à l'école primaire, au collège et au lycée, la création et l'organisation d'un enseignement de l'histoire des arts portant sur six domaines : arts de l'espace, du langage, du quotidien, du son, du spectacle vivant, et du visuel. Ce décret fait suite à la création d'un Haut conseil de l'éducation artistique et culturelle (2005), lui-même précédé en 2001 par le rapport Tasca-Lang sur les arts et la culture ${ }^{8}$. Il est complété par un Guide pour le parcours d'éducation artistique et culturelle (MEN, 2015b) qui rappelle les attendus du décret et propose des pistes d'applications concrètes 9 . On ne peut que se réjouir du fait que le texte officiel finisse par reconnaitre la validité des expériences théorisées et réalisées par des enseignants novateurs, comme en témoignent les nombreux articles de Pratiques qui favorisent le dialogue entre les disciplines et rendent compte de situations pédagogiques partenariales (ex. $\mathrm{n}^{\circ}$ 41, A. Petitjean, écriture et représentation d'une pièce de théâtre avec l'aide d'un metteur en scène; $\mathrm{n}^{\circ} 39, \mathrm{C}$. Masseron, fabrication d'une fresque à partir d'un travail commun français/dessin; ex. numéros 69 et 133-134 théorisant les expériences qui associent français et histoire). À quoi s'ajoutent les numéros qui, comme le prescrivent les textes officiels, rendent compte «d'activités qui développent la créativité de l'élève ». Voir les numéros consacrés respectivement aux ateliers d'écriture $\left(\mathrm{n}^{\circ} 61\right)$ et à l'écriture d'invention ( $\left.n^{\circ} 127-128\right)$. Sans négliger pour autant les numéros portant sur les capacités d'analyse des œuvres: voir les nombreux numéros consacrés au commentaire, à l'interprétation des textes mais aussi à l'analyse de l'image $\left(n^{\circ} 7-8, n^{\circ}\right.$ 18-19).

3 Ce sont ces publications passées et ces changements institutionnels récents qui ont justifié principalement la parution de ce numéro de Pratiques intitulé «Didactiques et médiations des Arts et de la Littérature ». Il résulte d'un séminaire pluridisciplinaire qui s'est déroulé dans le cadre du Centre de recherche sur les médiations (Crem) et a été ouvert à des intervenants extérieurs qui ont enrichi notre recherche.

Le numéro est subdivisé en deux parties selon que les articles portent sur la mise en relation entre l'enseignement $d u$ français et des autres disciplines (partie I) ou des rapports entre école et musées dans le développement des compétences culturelles des élèves (partie II).

5 Auparavant, nous avons retenu la description que fait Sylvie Octobre, à la suite des enquêtes d'O. Donnat $(1998,2003,2008)$ ou des articles réunis par de D. Pasquier (2005), des comportements culturels des enfants et des adolescents en régime «technoculturel ». Elle constate une présence grandissante des techno-cultures (smartphones, tablettes numériques, films en streaming, vidéos...) dans les environnements enfantins et juvéniles. Ce qui a pour conséquence une modification des attentes à l'égard des secteurs de la culture classique et des changements dans les modes de consommation culturelle. Parmi les normes comportementales les plus caractéristiques, on trouve la valorisation de l'individualisme et de l'expression de soi, de l'implication émotionnelle, de l'immédiateté et du divertissement. À quoi s'ajoute le primat donné à la «mobilité » 
sous des formes diverses (zapping des programmes, métissage des genres, cosmopolitisme esthétique et culturel). La figure du professionnel-amateur est assez représentative de ce désir d'implication active (production et réception) dans les contenus d'un monde culturel en permanente mutation. Ce qui a pour effet de provoquer des discours de déploration à propos d'une perte de la qualité culturelle, une volonté de contrôle par une pédagogisation des activités de loisir et des interrogations à propos de la place et des fonctions des médiations éducatives. L'intérêt de l'article est de mieux faire connaitre l'état des usages culturels des élèves en dehors de la sphère scolaire, et du même coup, d'aider à mieux comprendre leurs réactions face aux œuvres (tableaux ou romans) que certains articles du numéro décrivent.

\section{Pour une interdidactique de la littérature et des arts}

6 Nous n'avons pas l'intention de revenir sur l'abondante littérature qui existe à propos de la didactique en tant que champ disciplinaire, ses concepts fondamentaux (Reuter et al., 2007) et les conflits frontaliers entre didactique et pédagogie (Astolfi, 1997 ; Astolfi \& Houssaye, 1996 ; Develay, 1997) ni sur la crise identitaire du français partagé de plus en plus entre langue et littérature (Daunay, 2010 ; Petitjean, 2014). Nous n'insisterons pas plus sur les principales différences entre la didactique du français (Halté, 1992; Marquilló Larruy, 2001 ; Rosier, 2002 ; Daunay \& Reuter, 2008) et la didactique des arts (Chabanne, 2016). Il nous faut, par contre, nous arrêter un instant sur ce qu'implique la mise en relation entre deux disciplines (les arts et la littérature) que préconisent les textes officiels tels qu'Organisation de l'enseignement de l'histoire des arts. École, Collège, Lycée (MEN, 2008) instaurant ce nouvel enseignement à l'école primaire et au collège, le Parcours d'éducation artistique et culturelle (MEN, 2015b) ou les plus récents Enseignements pratiques interdisciplinaires (Éduscol, 2015). Ces textes, en particulier celui consacré à l'histoire des arts, posent des problèmes tant épistémologiques que pratiques et soulèvent des questions, comme l'ont bien montré tant S. Fabre (2013) que J.C. Chabanne $(2013,2016)$. Sans avoir les moyens ni le recul suffisant pour mesurer les effets de ce nouveau domaine d'enseignement, on ne peut que rappeler certaines questions fondamentales: quel est le statut de cette discipline aux frontières incertaines alors qu'elle se veut au carrefour des autres disciplines? Quels types de relations pluri-, inter-, trans- disciplinaires et interdidactiques impliquent sa mise en œuvre dans les classes et sa problématisation au niveau des recherches didactiques? En fonction de quelles pratiques sociales de référence définir la posture d'«amateur éclairé » et le type de discours que les élèves sont censés produire ? Comment évaluer la compétence des élèves quand on attend d'eux qu'ils sachent "parler de façon sensible d'œuvres d'art »?

7 Selon les articles du présent numéro, des éléments de réponse sont apportés à ces questions, que l'on se place au niveau de la formation des maitres, des textes officiels et des manuels ou des pratiques dans les classes.

Dans son article qui ouvre le numéro, Sylviane Ahr rappelle que, ces dernières années, nombreux sont les discours institutionnels qui prescrivent de «mettre en relation » et « de faire dialoguer » les œuvres littéraires et les autres créations artistiques. Au niveau des collèges et des lycées, sont privilégiées les œuvres iconographiques et cinématographiques, sans délaisser pour autant les représentations théâtrales. Pour $\mathrm{S}$. Ahr, il était donc opportun d'observer en quoi la formation initiale tient compte de 
ces directives. En prenant pour exemple deux épreuves orales d'admission du Capes de Lettres modernes, elle s'est livrée à l'analyse des rapports de jury des trois dernières sessions (2014-2016). Pour la première épreuve orale (épreuve de mise en situation professionnelle), il est demandé de traiter un dossier composé d'un texte littéraire et d'un document iconographique. Même si, dans tous les cas, l'analyse de l'image doit servir à conforter l'étude du texte donné à étudier, il ressort que deux orientations sont possibles. Soit le document est susceptible d'apporter des informations contextuelles sur le texte ou d'ouvrir à des relations intertextuelles et interartistiques. Soit l'accent est mis sur le travail interprétatif (c'est le cas en particulier des pratiques adaptatives, de mises en scènes ou d'illustrations) mené par l'auteur du texte cible par rapport au texte source dont les œuvres relèvent de sémiotiques hétérogènes. Pour la seconde épreuve (analyse d'une situation professionnelle), le dossier proposé, selon les options Langue et littératures françaises, Théâtre, Cinéma) est composé de textes et de documents complémentaires (tableaux, photographies, bandes dessinées, captations théâtrales, séquences filmiques). Même s'ils sont bien en deçà, en terme d'interarticité, par rapport aux textes officiels, les attendus de l'épreuve représentent une certaine ouverture aux différentes " formes d'expression artistique ». Dans une seconde partie, $\mathrm{S}$. Ahr revient sur les directives institutionnelles qui prescrivent de favoriser en classe la rencontre sensible avec les œuvres artistiques et précisent qu'il convient que les élèves sachent parler des œuvres en conjuguant «dimension sensible et dimension rationnelle». Cette tension entre rapport impliqué ("émotion esthétique») et distancié («jugement critique ») par rapport aux œuvres se retrouve dans les différents rapports du concours et nécessite que soient menées des recherches didactiques susceptibles de résoudre certaines contradictions.

9 Marie-Sylvie Claude commence par rappeler que la « lecture de l'image » fait partie du cahier des charges du professeur de français puisqu'il est question d'acquérir des "éléments d'analyse de l'image ", de "fréquenter les œuvres d'art» et d'aborder le «dialogue entre les arts». En fonction de quoi elle s'intéresse aux rapports entre littérature et peinture du point de vue des élèves selon qu'ils ont à commenter des textes littéraires ou des tableaux. Pour ce faire, M.-S. Claude établit un protocole d'enquête dans lequel elle croise un critère d'ordre sémiotico-herméneutique (texte/ image) et un critère socioculturel (type d'établissement). Elle obtient ainsi un corpus comprenant des écrits de 350 élèves de troisième et de seconde. Ils ont été invités à faire part de leurs réactions face à deux ensembles, l'un composé de trois textes et trois reproductions de tableaux du XXe siècle, l'autre, d'un nombre équivalent de textes et de tableaux du XVII ${ }^{e}$ siècle. À quoi s'ajoutent les propos qu'ils ont tenus lors d'entretiens post-passation. Cette recherche mobilise un outillage descriptif qui mixte les apports issus de la didactique du français (B. Daunay, D. Bucheton), des théories de la lecture (U. Eco, J.-L. Dufays), des sciences des arts (N. Goodman, E. Panofsky), de la sociologie de la culture (P. Bourdieu, S. Octobre) et de la sociologie des apprentissages (É. Bautier et P. Rayou). Théories qui sont souvent plus juxtaposées qu'intégrées dans l'analyse et auxquelles il manque les théories des représentations et de l'analyse de discours fort utiles, au demeurant, puisqu'il s'agit d'étudier ce que pensent les élèves de leurs rapports à la littérature et à la peinture. Néanmoins, on retiendra de cette enquête que les élèves se déclarent plus réceptifs à la peinture qu'à la littérature, et cela d'autant plus qu'ils relèvent de milieux socioculturels défavorisés. Cette impression de familiarité tient au fait que la peinture est plutôt accessible au niveau préiconographique (identification des éléments figurés et de leur valeur expressive). Mais 
comme ces élèves n'ont pas les références culturelles nécessaires à la compréhension iconographique, ils rencontrent des difficultés interprétatives tout aussi importantes qu'en littérature. M.-S. Claude montre aussi que l'investissement éthique des élèves est plus grand en littérature qu'en peinture. Elle en conclut qu'il est important d'établir un dialogue entre les deux arts, à condition que les enseignants amènent les élèves à identifier les éléments de réussite dans une activité pour les transposer dans l'autre.

10 Avec Nicole Biagioli, les relations entre les pratiques artistiques scolaires, qu'elle appelle «interartialité », sont analysées non pas du point de vue de la réception mais de la production. Ce parti pris est justifié par le fait que dans les pratiques sociales de référence, qu'elles relèvent de la communication ou des arts, nombreux sont les objets discursifs qui associent des sémiotiques hétérogènes. L'article est divisé en deux parties. Dans la première, N. Biagioli adopte une perspective historique qui lui permet, en référence aux programmes de français des collèges (1977-1995) et en s'appuyant sur les manuels, de retracer les moments-clés de la didactisation de l'interartialité, en particulier au niveau du traitement de l'image et des arts. Il apparait que dans les années 70 , au moment où, par le biais du thématisme, une certaine place est réservée à la créativité, on invite les élèves à créer des objets qui font appel, au-delà du dessin et de la musique, à des matières telles que la technologie, voire l'EPS. Avec la rénovation des collèges de 1995, la discipline français va accorder une place prépondérante au discursif qui se traduit par un apprentissage progressif des types textuels de la $6^{\mathrm{e}}$ à la $3^{\mathrm{e}}$ et un appauvrissement de la relation textes/images dû à un assujettissement de ces dernières aux logiques discursives. La réforme de 2008, en introduisant l'histoire des arts comme une matière fondamentalement interdidactique va substituer «l'étude de l'image » à la "lecture de l'image » et se traduire par des activités de production impliquant l'interartialité avec le risque de mésestimer l'importance des apprentissages langagiers. Dans la seconde partie de son article, N. Biagioli analyse deux pratiques de classes en collège liées à l'interartialité. L'une correspond à l'écriture d'un texte à partir de la première de couverture d'un roman étudié en classe, l'autre, à la fabrication d'objets emblèmes de leur lecture. Dans les deux cas, un questionnaire est fourni aux élèves portant sur la production, l'évaluation et la conscientisation de l'interartialité et un autre aux enseignants axés sur l'interartialité et ses conséquences sur les apprentissages. Au terme d'une analyse minutieuse des réponses, N. Biagioli achève son étude par une réflexion portant sur les intérêts et les difficultés des pratiques interartiales et sur la nécessité de leur didactisation.

11 Avec les contributions de C. Boutevin puis d'A. Leclaire-Halté et L. Maisonneuve, il s'agit d'étudier des œuvres qui relèvent du champ de la littérature de jeunesse et ont pour propriété d'associer texte et image.

12 C'est ainsi que Christine Boutevin s'arrête sur les «livres de poèmes illustrés » que l'on trouve dans les listes officielles pour les cycles 2 et 3. Dans un premier temps, elle se livre à une typologie de ces ouvrages qui associent langage verbal et plastique. Ce qui lui permet de classer ces livres de poèmes en quatre types (l'anthologie illustrée, le poème-album, le recueil illustré, l'album-poème) dont elle précise à chaque fois quelles sont les logiques éditoriales qui président à leur confection et comment est pensée, pour chaque type, la relation entre le verbal et le visuel. C. Boutevin insiste sur la nécessité de donner les moyens aux élèves d'acquérir les compétences requises pour la lecture et l'interprétation de ce type de poèmes. Dans un second temps, C. Boutevin rend compte d'un dispositif expérimenté en formation initiale dans le cadre du Master 
MEFF. Il s'agit de lire un album-poème, c'est-à-dire un type de livre problématique du point de vue générique et complexe au regard de l'interdépendance du texte et de l'image. Si l'on en juge par les réponses à un questionnaire, pour les étudiants à qui l'on demande leurs réactions face à ce type d'œuvre la prise en compte de l'interdépendance entre poème et image est problématique, l'iconique étant trop souvent pensé comme une simple illustration du verbal.

13 Anne Leclaire-Halté et Luc Maisonneuve, de leur côté, s'intéressent à la relation texte-image telle qu'elle se présente dans les albums documentaires consacrés aux peintres dans les ouvrages pour la jeunesse. Pour ce faire, ils ont choisi trois ouvrages consacrés à Matisse et plus particulièrement à la présentation du tableau La Danse. Dans une première partie, ils se livrent à une description du dispositif proposé par les trois albums sous la forme d'une double page comportant la reproduction du tableau de Matisse et un texte d'accompagnement. Il ressort de leur analyse que ce qui caractérise ces doubles pages est qu'elles associent l'image du tableau et un texte tout à la fois descriptif, interprétatif et subjectif, privilégiant, de ce fait, les émotions ressenties à la vue du tableau au détriment des informations contextuelles à propos de la production du tableau. Dans une seconde partie, A. Leclaire-Halté et L. Maisonneuve relatent et analysent une séquence réalisée dans une classe de $\mathrm{CM} 2$ au cours de laquelle l'enseignante poursuit un double objectif : la maitrise du lire/écrire et la sensibilisation aux œuvres d'art. Les élèves, après avoir observé des doubles pages d'album, ont été invités successivement à rédiger une description écrite du tableau puis à élaborer pour un site web de l'école une double page présentant le même tableau. On retiendra de cette expérience dont on suit les différentes étapes qu'il est possible de donner aux élèves l'occasion de s'exprimer sur une œuvre d'art et que c'est l'un des moyens de faire d'eux de futurs amateurs d'art.

L'adaptation en ce qu'elle est un processus de "transécriture» et de "transmodalisation » est un autre moyen d'envisager le dialogue entre littérature et arts. C'est ainsi qu'Armelle Hesse-Weber rend compte d'une expérience d'enseignement menée dans une classe de cinquième de collège au cours de laquelle une étude de La Farce de Maître Pathelin a été suivie par son adaptation en BD, avec l'aide des professeurs de français et d'arts plastiques. A. Hesse-Weber commence par constater que, dans le champ de la littérature de jeunesse, nombreuses sont les adaptations en $\mathrm{BD}$ de pièces du répertoire classique et justifie, après $\mathrm{H}$. Gondrand et A. Vibert (2008) la pratique scolaire de l'adaptation au nom d'intérêts tant didactiques (appropriation d'œuvres classiques du patrimoine, propédeutique aux notions de genre et de récit) que pédagogiques (motivation des élèves, développement des compétences de lecture et d'écriture). Dans un second temps, A. Hesse-Weber rend compte de manière détaillée et avec l'aide de tableaux en annexe, de l'étude intégrale de la farce puis de sa transposition en BD. Cette expérience qui repose sur un travail en projet et une concertation interdisciplinaire, permet indéniablement de développer les compétences liées à la maitrise du français (écriture, lecture, langue et oral) tout en favorisant l'éducation artistique et culturelle.

15 André Petitjean, pour sa part, tenant compte des programmes officiels de 2010 pour les classes de L des lycées ainsi que de l'épreuve professionnelle du Capes concernant les options "Langue et littératures françaises, Théâtre, Cinéma", se livre à une réflexion à propos de l'adaptation théâtrale en prenant comme exemple, l'adaptation par J. Tardieu du Candide de Voltaire. Après avoir rappelé que l'adaptation est une 
pratique courante que l'on retrouve dans tous les arts, il commence par définir à grands traits ce qui caractérise cette forme de réécriture. Suite à quoi, dans une première partie, il précise certaines caractéristiques de l'œuvre de Voltaire (formes et contenus) afin d'étudier, dans ce jeu de tension entre conservation et transformation qu'implique toute adaptation, le parti pris esthétique et idéologique de J. Tardieu. Dans une seconde partie, A. Petitjean s'attache à décrire les modifications discursives et stylistiques que J. Tardieu apporte au texte de Voltaire. Ce faisant, il montre en quoi toute adaptation implique une activité interprétative qui est à penser comme un mouvement intentionnel de décontextualisation/recontextualisation, une dialectique entre un dire et un redire et une tension entre conservation et innovation.

Isabelle De Peretti, de son côté, réfléchit à la façon dont le théâtre en situation scolaire, sauf à limiter son approche à la seule lecture des textes, est un objet nécessairement interdidactique. Cela tient au fait qu'il est un art «à double régime d'immanence » (texte et représentation) impliquant deux régimes de réception ou de lecture, l'une centrée sur la textualité dramatique, l'autre sur la textualité scénique. Il s'en suit qu'I. De Peretti, en s'appuyant sur les théoriciens du théâtre ainsi que sur les principales revues et collections de didactique du français, propose que l'accent soit mis sur la mise en voix des textes au cours de l'étude des textes dramatiques. Elle montre que le fait de devoir vocaliser un texte de théâtre modifie les modalités de sa lecture. À ce niveau, l'intervention du professeur d'éducation musicale ou d'un comédien s'impose. Cet investissement du texte par la voix est pour I. De Peretti d'autant plus justifié que le répertoire contemporain, en particulier destiné à la jeunesse, est "un théâtre des voix». L'utilisation du jeu dramatique est tout aussi nécessaire à la lecture du texte de théâtre tant pour la compréhension du texte que pour son anticipation scénique.

\section{L'expérience esthétique entre classe et musée}

Nous avons fait l'économie d'un article consacré à la " médiation culturelle » à propos des arts (Dufrêne \& Gellereau, 2010 ; Lamizet, 1999 ; Heinich, 2009; Iborra-Sanchez, 2014 ; Caune, 2017) et de la littérature (Privat \& Reuter, 1990 ; Soumagnac, 2008), tant le sujet a été traité d'un point de vue aussi bien sociologique, épistémique, éthique, esthétique que didactique. Pour ne prendre qu'un exemple, on peut se reporter aux parutions dirigées par J.-P. Dufiet (2012 et 2014) qui traitent, à travers la «visite guidée » des aspects tant institutionnels, interactionnels que discursifs de la médiation culturelle dans le cadre des musées. Disons qu'entre l'œuvre et le public (spectateurs ou lecteurs), il existe toute une chaine d'intermédiaires sous la forme d'institutions, d'acteurs, de dispositifs, et de discours. C'est pourquoi, il nous a semblé important de poursuivre la réflexion sur les modalités de médiation des objets d'arts selon qu'elles ont lieu dans les musées et en situation scolaire.

18 L'article de Christine Deronne, qui ouvre la seconde partie du numéro, est issu d'une recherche menée dans le cadre d'un Lieu d'éducation associé (LéA), réseau de l'Institut français d'éducation (IFÉ) qui fédère une trentaine de groupes de recherche collaborative. Les trois parties de cet article traitent de la réception de l'œuvre d'art par des élèves de l'école primaire, réception dont l'auteure souhaite qu'elle concilie à la fois l'expression des émotions et l'investissement intellectuel. L'œuvre picturale n'ayant véritablement un statut artistique que dans un musée ou une galerie, l'article 
s'intéresse au parcours d'une classe en visite au musée. Dans un premier temps, C. Deronne définit les spécificités de l'espace muséal par rapport à l'espace scolaire, ainsi que la place de ses acteurs, les élèves, l'enseignant et le médiateur. Elle met l'accent sur les tensions et les interrogations liées à la rencontre avec l'œuvre d'art dans cet espace, et définit les conditions d'une médiation réussie. Dans une seconde partie, certaines distinctions sont tentées, entre émotion ordinaire et esthétique, entre plaisir esthétique et artistique, entre réception informée et non informée, entre jugement de gout et jugement esthétique. En effet, préciser ces termes est important car ils sont susceptibles d'aider les enseignants à clarifier les dispositifs mis en place pour favoriser la rencontre avec l'œuvre. Enfin, trois situations didactiques, lors des visites de deux expositions, sont évoquées, toutes trois visant à favoriser l'émergence de la parole des élèves sur l'œuvre. Ces situations de paroles peuvent permettre à l'enseignant de voir où se situent les élèves, entre appréhension sensorielle et appréhension plus intellectuelle, et de leur proposer des situations les amenant à une émotion réfléchie.

Il est aussi question de langage dans l'article Jean-Charles Chabanne et al. qui porte sur les gestes professionnels langagiers. Intitulés « Autour de la sculpture et du tactile : jeux de langage(s), conduites d'élèves et arts de faire professionnels en cycle 1 » ces travaux sont issus d'une recherche menée au sein d'un LéA, le LéA MusÉcoleMontpellier, qui se donne pour objectif de concevoir des ressources numériques de formation aux gestes professionnels liés à la rencontre avec des œuvres d'art, en établissant un lien entre ce qui se joue dans un lieu d'exposition, et les apprentissages scolaires. L'article livre une réflexion conduite à partir d'une exposition au Musée Fabre de Montpellier, qui place le toucher au cœur de la découverte de l'art. Cette exposition, en effet, a proposé au public une galerie de sculptures à découvrir tactilement. L'équipe du LéA s'interroge alors sur une médiation possible auprès d'élèves de maternelle: quels sont les contenus éducatifs potentiels liés à cette exposition? Dans quelles logiques professionnelles se situent les divers membres de l'équipe? C'est ainsi que certains gestes professionnels langagiers sont dégagés : la présentation des savoirs et ses moments opportuns, l'écoute et la gestion des jeunes élèves, dans leurs verbalisations mais aussi leur gestuelle, leurs regards, la question du lexique, autant de facteurs qui sont au cœur des interrogations soulevées et des choix proposés.

L'article d'Elisa Ravazzolo qui suit a pour objectif de caractériser le discours des médiateurs de musée, en étudiant les stratégies discursives et les ressources interactionnelles que ces derniers mettent en place auprès d'enfants de l'école élémentaire. Il se fonde sur l'analyse d'un corpus oral constitué à partir de l'enregistrement audio et vidéo de deux visites guidées au Musée d'art et d'histoire de Saint-Denis, conduites par deux médiatrices auprès d'un groupe de quatre enfants de $\mathrm{CP}$ malvoyants et d'un CE1. Contrairement à la position de C. Deronne qui définissait la médiation non comme une question de statut, mais d'objectif et de modalités, et qui défendait l'idée qu'un enseignant pouvait être médiateur, E. Ravazzolo distingue bien le rôle du médiateur, qui est de faire savoir, dans un climat détendu, de celui de l'enseignant, qui est de faire apprendre. L'article décrit d'abord la situation d'interaction dans laquelle se trouve le médiateur, caractérisée par la position haute de ce dernier. Il souligne l'importance des interactions avec les enfants car elles sont révélatrices de l'investissement de ces derniers dans la visite, leurs interprétations des œuvres présentées, leur appropriation des connaissances transmises... Après cette 
évocation de la situation d'interaction, l'article énumère des stratégies de transmission du savoir auxquelles les médiatrices du corpus ont recours, qu'il s'agisse de la modalité d'introduction des objets muséaux, de la façon dont ils sont expliqués, ou du recours au questionnement qui caractérisent leur discours.

21 L'article de Françoise Rigat concerne également les pratiques non pas scolaires, mais muséales, en analysant une autre forme de médiation. Plus spécifiquement, l'auteure s'interroge sur le type de médiation présent dans les livrets pour enfants, aujourd'hui très fréquemment mis à leur disposition par les musées pour les accompagner dans la visite des expositions. Elle rappelle d'abord que la médiation culturelle s'est construite contre une vision du rapport au savoir comme transmission de connaissances, au profit «du plaisir lié à la culture, à l'expérience, à la sociabilité ». Qu'en est-il alors des informations données dans ces livrets destinés à un jeune lectorat? À partir d'un corpus de cinq livrets choisis pour leur diversité mais aussi pour leur rigueur de conception, F. Rigat fournit cinq exemples de cette médiation. Dans une approche qui prend en compte la mise en page aussi bien qu'une analyse de contenu discursive et énonciative, elle montre la diversité de cette production, tantôt dans la proximité avec un modèle scolaire, didactique et plutôt descendant, tantôt valorisant le ludique comme accès principal à l'œuvre et/ou au musée. Le dernier exemple cité, celui d'un livret qui s'adresse à l'enfant, mais aussi à sa famille, illustre les difficultés à sortir des rôles habituels et à faire jouer à l'adulte le rôle d'accompagnateur de l'enfant plutôt que l'inverse. L'article se conclut sur le fait que nombre de ces fascicules, qui à l'origine se donnaient pour but d'initier à l'art, sont finalement plus destinés à un monde d'initiés. Pour son auteure, le moyen de démocratiser ce dernier tient à la langue utilisée : l'attachement à une langue soutenue, fréquente dans ces livrets, peut être inhibiteur, alors que, sans sacrifier au contenu, leurs concepteurs pourraient se mettre langagièrement beaucoup plus à la portée des enfants.

Pour finir, Cora Cohen-Azria et Ana Dias-Chiaruttini traitent de la médiation à propos d'une exposition au musée d'histoire naturelle de Lille conçue à partir de l'album de littérature de jeunesse De la petite taupe qui voulait savoir qui lui avait fait sur la tête. Leur article se déroule en trois temps. Il propose d'abord une double lecture de l'album, par une didacticienne du français et une didacticienne des sciences, et montre comment ces deux lectures s'enrichissent l'une l'autre. Il se consacre ensuite à la scénographie de l'exposition, qui comble les inférences du texte et des images de l'album: la fiction est convoquée, mais pour permettre d'aborder des questions scientifiques, qui sont dans l'implicite de la fiction. Enfin, il analyse le discours d'une guide assurant la visite de cette exposition par une classe, en s'interrogeant sur la place qu'elle donne à l'album et sur les caractéristiques du discours de médiation scientifique. La conclusion est que la scénographie s'inspire de la fiction en accordant une place centrale à l'enquête scientifique suggérée par l'album, et que la médiation réintroduit la fiction. L'exposition, en fait, invite ses visiteurs à une double lecture, tant fictionnelle que scientifique, de l'album.

Le numéro se referme avec un dossier, coordonné par A. Rabatel et C. Masseron, sur " la question du prédicat». Les contributions retracent l'historique d'une polémique alimentée par les médias et discutent, au plan épistémologique et didactique, du bienfondé de cette notion de prédicat introduite dans les instructions de 2015. La discussion didactique s'appuie sur les expériences de la francophonie (France, Québec, Suisse 
romande et Wallonie) et examine les enjeux de formation (des maitres et des élèves) qui sont impliqués.

\section{BIBLIOGRAPHIE}

AStolfi, J.-P. (1997). « Du “tout" didactique au "plus" didactique ». Revue française de pédagogie 120, p. 67-73. En ligne : http://www.persee.fr/doc/rfp_0556-7807_1997_num_120_1_1157.

ASTOLFI, J.-P. \& housSAYE, J. (1996). « Didactique et pédagogie sont dans un bateau ». Éducations 7, p. 34-42.

BEST, F. (1973). Pour une pédagogie de l'éveil. Paris : A. Colin.

BOURDIEU, P. \& GROS, F. (1989). Principes pour une réflexion sur les contenus de l'enseignement. Paris : Ministère de l'Éducation nationale.

CAHIERS PÉDAGOGIQUES. (1971). « Liaisons interdisciplinaires ». 97.

CAHIERS PÉDAGOGIQUES. (1974). « L'équipe éducative ». 123.

CAHIERS PÉDAGOGIQUES. (1976). « Coordination des disciplines ». 148-149.

CAHIERS PÉDAGOGIQUES. (1978). « Apprendre sans cloisons ». 166.

CAUNE, J. (2017). La médiation culturelle. Expérience esthétique et construction du Vivre-ensemble. Grenoble : Presses universitaires de Grenoble.

CHABANNE, J.-C. (2013). « Histoire des arts et jeux de langage : comment parler de l'œuvre ». Le Français aujourd'hui 182, p. 55-66. En ligne : https://www.cairn.info/revue-le-francais-aujourdhui-2013-3-page-55.htm.

CHABANNE, J.-C. (2016). « Approches interdisciplinaires des enseignements culturels et artistiques dans le cadre de l'enseignement de l'histoire des arts : un modèle complexe, une didactique impossible ?» Tréma 45, p. 59-69. En ligne : https://trema.revues.org/3501.

DAUNAY, B. (2010). « Français et littérature : une ou des discipline(s) ». Le Français aujourd'hui 168, p. 23-30. En ligne : https://www.cairn.info/revue-le-francais-aujourd-hui-2010-1-page-23.htm.

DAUNAY, B. \& REUTER, Y. (2008). « La didactique du français : questions d'enjeux et de méthodes ». Pratiques 137-138, p. 57-78. En ligne : https://pratiques.revues.org/1152.

DEVELAY, M. (1997). « Origines, malentendus et spécificités de la didactique ». Revue française de pédagogie 120, p. 59-66. En ligne : http://www.persee.fr/doc/

rfp_0556-7807_1997_num_120_1_1156/.

DOMENACH, J.-M. (1989). Ce qu'il faut enseigner. Paris : Seuil.

DONNAT, O. (1998). Les pratiques culturelles des français. Paris : La Documentation française.

DONNAT, O. (dir.) (2003). Regards croisés sur les pratiques culturelles. Paris : La Documentation française. 
DONNAT, O. (dir.) (2008). Les pratiques culturelles des français à l'ère numérique. Paris : La Découverte/ Ministère de la Culture et de l'Éducation.

DUFIET, J.-P. (2012). Les visites guidées. Discours, interaction, multimodalité. Trento : Università degli Studi di Trento. Dipartimento di Studi Letterari, Linguistici e Filologici.

DUFIET, J.-P. (2014). L'objet d'art et de culture à la lumière de ses médiations. Trento : Università degli Studi di Trento. Dipartimento di Studi Letterari, Linguistici e Filologici.

DUFRÊNE, B. \& GELLEREAU, M. (2010). « La médiation culturelle : enjeux professionnels et politiques ». In : Liquète, V. (dir.), Médiations. Paris : CNRS Éd., p. 67-83.

ÉDUSCOL. (2015). Enseignements pratiques interdisciplinaires. En ligne : http://eduscol.education.fr/ cid99750/epi.html.

FABRE, S. (2013). «L'histoire des arts et le temps à l'œuvre ». Le Français aujourd'hui 182, p. 31-42. En ligne : https://www.cairn.info/revue-le-francais-aujourd-hui-2013-3-page-31.htm.

FINKIELKRAUT, A. (1987). La défaite de la pensée. Paris : Gallimard.

GONDRAND, H. \& VIBERT, A. (éds) (2008). Adapter des œuvres littéraires pour les enfants. Enjeux et pratiques scolaires. Grenoble : SCEREN-CRDP de l'Académie de Grenoble.

HALTÉ, J.-F. (coord.) (1982). « Travailler en projet ». Pratiques, 36.

HALTÉ, J.-F. (coord.) (1992). La didactique du français. Paris : Presses universitaires de France.

HEINICH, N. (2009). Faire voir. L'art à l'épreuve de ses médiations. Paris : Les Impressions nouvelles.

IBORRA-SANCHEZ, C. (2014). La médiation face à l'école : vers une typologie des médiateurs culturels dans les Musées et Centres d'art. Thèse en sciences de l'éducation : Université Lumière Lyon 2.

LA MARTINIÈRE, D. de (1984). Lettre ouverte à tous les parents qui refusent le massacre de l'enseignement. Paris : A. Michel.

LAMIZET, B. (1999). La médiation culturelle. Paris : L'Harmattan.

LE FRANÇAIS AUJOURD’HUI. (1979). « Pratiques interdisciplinaires ». 45.

LE FRANÇAIS AUJOURD'HUI. (1981a). « Collèges 80 ». 53.

LE FRANÇAIS AUJOURD'HUI. (1981b). « Français/Philo ». 56.

LE FRANÇAIS AUJOURD'HUI. (1982). « Des classes de français ». 59.

LE FRANÇAIS AUJOURD’HUI. (1986). « Le Français au carrefour des disciplines ». 74.

LEGRAND, L. (1981). Pour un collège démocratique. Rapport au ministre de l'Éducation nationale. Paris : La Documentation française.

LENOIR, Y. \& SAUVE, L. (1998). « De l'interdisciplinarité scolaire à l'interdisciplinarité dans la formation à l'enseignement : un état de la question ». Revue française de pédagogie 125, p. 109-146. En ligne : http://www.persee.fr/doc/rfp_0556-7807_1998_num_125_1_1111.

MARQUILló LARRUY, M. (éd.) (2001). Questions d'épistémologie en didactique du Français. Poitiers : Forell.

MASCHINO, M. (1984). Voulez-vous vraiment des enfants idiots? Paris : Hachette.

MEIRIEU, Ph. (1998). Quels savoirs enseigner dans les lycées ? Paris : La Documentation française. 
MINISTÈRE DE L'ÉDUCATION NATIONALE (2001). Le plan pour les arts et la culture. Paris : Centre national de documentation pédagogique.

MINISTÈRE DE L'ÉDUCATION NATIONALE (2006). Socle commun de connaissances et de compétences. Décret $\mathrm{n}^{\circ}$ 2006-830, 11-7-2006, Journal officiel du 12-7-206.

MINISTÈRE DE L'ÉDUCATION NATIONALE (2008). Organisation de l'enseignement de l'histoire des arts. École, Collège, Lycée. Bulletin officiel $\mathrm{n}^{\circ} 32,28$ août 2008.

MINISTÈRE DE L'ÉDUCATION NATIONALE (2010). Programme d'enseignement obligatoire au choix d'arts en classe de première littéraire, d'enseignement de spécialité au choix d'arts en classe terminale littéraire et d'enseignement facultatif d'arts au cycle terminal des séries générales et technologiques. Bulletin officiel spécial nº 9, 30 septembre 2010.

MINISTÈRE DE L'ÉDUCATION NATIONALE (2015a). Socle commun de connaissances, de compétences et de culture. Décret n $n^{\circ}$ 2015-372 du 31-3-2015, Bulletin officiel nº 17, 23 avril 2015.

MINISTÈRE DE L'ÉDUCATION NATIONALE (2015b). Guide pour le parcours d'éducation artistique et culturelle. Paris : La Documentation française.

MORIN, E. (1998). Pourquoi et comment articuler les savoirs. Paris : La Documentation française.

PASQUIER, D. (2005). Cultures lycéennes. Paris : Éd. Autrement.

petitjean, A. (coord.) (1981). « Travail en équipe ». Pratiques, 31.

PETITJEAN, A. (coord.) (2014). « 40 ans d'histoire de la "lecture littéraire" au secondaire à partir de la revue Pratiques ». Pratiques 161-162. En ligne : http://pratiques.revues.org/2155.

PRIVAT, J.-M. \& REUTER, Y. (éds) (1990). Lectures et médiations culturelles. Lyon, Presses universitaires de Lyon.

REUTER, Y. et al. (2007). Dictionnaires des concepts fondamentaux des didactiques. Bruxelles : De Boeck. ROSIER, J.-M. (2002). La didactique du français. Paris : Presses universitaires de France.

SOUMAGNAC, K. (2008). « La construction de la médiation littéraire sur internet : vers un changement de paradigme des pratiques d'écriture ». Études de communication 31, p. 175-188. En ligne : http://journals.openedition.org/edc/796.

WAYSBORD-LOING, H. (2000). L'image dans l'enseignement des lettres. Rapport de l'inspection générale. Ministère de la Jeunesse, de l'Éducation nationale et de la Recherche.

\section{NOTES}

1. Le rapport insiste sur l'intérêt des «activités interdisciplinaires (qui) ont pour but essentiel d'aider les adolescents à construire, de manière personnelle et motivée, leur propre savoir et à en appréhender le sens ».

2. Dans le sixième principe, il est conseillé de «favoriser les enseignements donnés en commun par des professeurs de différentes spécialités et même de repenser les divisions en "disciplines" ".

3. Il plaide, au nom de "l'inter-trans-poly-disciplinarité », pour le décloisonnement des savoirs au lycée. Il affirme clairement que «la mission première de l'enseignement est d'apprendre à relier » et en même temps « à problématiser ».

4. «Le lycée favorise l'approche interdisciplinaire ou pluridisciplinaire d'objets complexes ainsi que de questions ou problèmes qui figurent au programme de plusieurs disciplines. 
L'intervention de deux enseignants, de disciplines différentes, avec un même groupe d'élèves peut être organisée dans cette perspective. Cette possibilité offerte aux enseignants est prise en compte dans leur service ».

5. Dans un rapport de l'inspection générale de Lettres de 1999-2000, H. Waysbord-Loing (2000) faisant allusion aux différents dispositifs regrettait que «Le travail en équipe disciplinaire ou pluridisciplinaire demeure encore restreint, malgré les nouvelles modalités de travail y incitant : parcours diversifiés ou travaux croisés en collège ; modules en lycée, et à partir de la rentrée 2000, les TPE, bien reçus par les enseignants, même si une définition plus précise du cadre de travail est demandée. On le constate, le travail pluridisciplinaire fait appel à des affinités personnelles plus qu'il ne résulte d'habitudes acquises entre disciplines. La collaboration entre littéraires et historiens est la plus fréquente. À ce titre, on peut regretter qu'au niveau du lycée les concordances de programmes existant pour le collège, n'aient pas été poursuivies. Pour dépasser le plan des contenus et accéder à l'analyse du langage visuel, le travail avec les collègues d'Arts plastiques apporte la complémentarité voulue. Il est limité par la faible disponibilité de ces enseignants qui ont un horaire très dispersé entre de nombreuses classes. Lorsqu'il existe de façon affirmée - par exemple au lycée de Valbonne (Nice) - le bénéfice est considérable. Au plan disciplinaire, il existe maintenant des échanges plus nombreux entre les enseignants de lettres, réputés individualistes. L'apparition d'un travail d'équipe, à tout le moins d'une réflexion commune, a été suscitée par la diversification des modalités de l'apprentissage (modules, aide individualisée, etc.) pour mieux répondre à la diversité des publics. »

6. «Le socle commun s'organise en sept compétences: la maîtrise de la langue française, la pratique d'une langue vivante étrangère, les compétences de base en mathématiques et la culture scientifique et technologique, la maîtrise des techniques usuelles de l'information et de la communication, la culture humaniste, les compétences sociales et civiques, l'autonomie et de l'initiative des élèves [...]. La culture humaniste contribue à la formation du jugement, du gout et de la sensibilité.

Elle enrichit la perception du réel, ouvre l'esprit à la diversité des situations humaines, invite à la réflexion sur ses propres opinions et sentiments et suscite des émotions esthétiques. Elle se fonde sur l'analyse et l'interprétation des textes et des œuvres d'époques ou de genres différents. Elle repose sur la fréquentation des œuvres littéraires (récits, romans, poèmes, pièces de théâtre), qui contribue à la connaissance des idées et à la découverte de soi. Elle se nourrit des apports de l'éducation artistique et culturelle.»

7. Domaine $1:$ «Comprendre, s'exprimer en utilisant les langages des arts et du corps. Sensibilisé aux démarches artistiques, l'élève apprend à s'exprimer et communiquer par les arts, de manière individuelle et collective, en concevant et réalisant des productions, visuelles, plastiques, sonores ou verbales notamment. Il connaît et comprend les particularités des différents langages artistiques qu'il emploie. Il justifie ses intentions et ses choix en s'appuyant sur des notions d'analyse d'œuvres ». Domaine 5 : «L'élève imagine, conçoit et réalise des productions de natures diverses, y compris littéraires et artistiques. Pour cela, il met en œuvre des principes de conception et de fabrication d'objets ou les démarches et les techniques de création. Il tient compte des contraintes des matériaux et des processus de production enrespectant l'environnement. Il mobilise son imagination et sa créativité au service d'un projet personnel ou collectif. Il développe son jugement, son goût, sa sensibilité, ses émotions esthétiques ».

8. «Aux trois niveaux du cursus scolaire, École primaire, Collège, Lycée, l'histoire des arts instaure des situations pédagogiques pluridisciplinaires et partenariales. Son enseignement implique la constitution d'équipes de professeurs réunis pour une rencontre, sensible et réfléchie, avec des œuvres d'art de tout pays et de toute époque. Il est aussi l'occasion de renforcer, autour d'un projet national conjoint, le partenariat entre les milieux éducatifs et les milieux artistiques et culturels. À travers lui, l'institution scolaire reconnait l'importance de l'art dans l'histoire des pays, des cultures et des civilisations. » 
9. En voici quelques exemples : «Visite du musée. À partir des éléments de connaissance et de l'approche sensible, préparation en classe de la présentation des œuvres dans le musée lors de la déambulation des élèves spectateurs »; "Découverte d'un album et de l'enchaînement entre les pages »; « Atelier de jeu théâtral conjointement par le professeur et le praticien », " présentation d'une petite forme dans d'autres classes ou dans un théâtre »; "Sortie théâtrale suivie d'un travail d'analyse de la représentation »; « Atelier d'écriture d'un conte », etc.

\section{AUTEURS}

\section{ANNE LECLAIRE-HALTÉ}

Université de Lorraine, CREM, EA 3476, ESPÉ de Lorraine, F-57000, France

\section{ANDRÉ PETITJEAN}

Université de Lorraine, CREM, EA 3476, F-57000, France 